\title{
337.
}

\section{NOTE SUR LA RÉALITÉ DES RACINES D'UNE ÉQUATION QUADRATIQUE.}

[From the Journal für die reine und angewandte Mathematik (Crelle), tom. LXI. (1863), pp. $367-368$.

A PRopos du mémoire que vient de publier M. Hesse (voir ce Journal t. LX., p. 305) je remarque que si l'une ou l'autre des deux formes

$$
(a, b, c, f, g, h \gamma)^{2}, \quad\left(a^{\prime}, b^{\prime}, c^{\prime}, f^{\prime}, g^{\prime}, h^{\prime} \chi\right)^{2}
$$

est une forme définie (forme qui conserve toujours le même signe pour des valeurs réelles quelconques des variables), l'équation suivante en $\lambda$ :

$$
\left|\begin{array}{cccc}
a-\lambda a^{\prime}, & h-\lambda h^{\prime}, & g-\lambda g^{\prime}, & x \\
h-\lambda h^{\prime}, & b-\lambda b^{\prime}, & f-\lambda f^{\prime}, & y \\
g-\lambda g^{\prime}, & f-\lambda f^{\prime}, & c-\lambda c^{\prime}, & z \\
x, & y, & z
\end{array}\right|=0
$$

aura ses deux racines réelles. En écrivant

$$
\begin{aligned}
& A=b c-f^{2}, \quad A^{\prime}=b^{\prime} c^{\prime}-f^{\prime 2}, \quad A_{1}=b c^{\prime}+b^{\prime} c-2 f f^{\prime} \\
& B=c a-g^{2}, \ldots
\end{aligned}
$$

de manière que $(A, B, C, F, G, H \chi)^{2}$ dénote la forme adjointe (ou réciproque) de $(a, b, c, f, g, h \gamma)^{2}$, cette équation prend la forme

$$
(A, \ldots \nmid x, y, z)^{2}-\lambda\left(A_{1}, \ldots \chi \chi x, y, z\right)^{2}+\lambda^{2}\left(A^{\prime}, \ldots \chi x, y, z\right)^{2}=0,
$$

et les racines étant réelles, on doit avoir

$$
\square=-4\left(A, \ldots \nmid(x, y, z)^{2}\left(A^{\prime}, \ldots \nmid(x, y, z)^{2}+\left[\left(A_{1}, \ldots \chi x, y, z\right)^{2}\right]^{2}=+.\right.\right.
$$


Or pour démontrer directement cette proposition, il n'est pas ce me semble possible d'exprimer $\square$ comme une somme de carrés; on a besoin de considérer une forme plus génerale, savoir une somme de carrés multipliés chacun par un coefficient litéral positif. Par exemple, en ne faisant attention qu'au coefficient de $x^{4}$, on doit avoir

$$
\square_{0}=-4\left(b c-f^{2}\right)\left(b^{\prime} c^{\prime}-f^{\prime 2}\right)+\left(b c^{\prime}+b^{\prime} c-2 f f^{\prime}\right)^{2}=+.
$$

Pour en faire la démonstration, on peut exprimer $\square_{0}$ sous la forme

$$
\square_{0}=\left(b c^{\prime}-b^{\prime} c\right)^{2}+4\left(b f^{\prime}-b^{\prime} f\right)\left(c f^{\prime}-c^{\prime} f\right),
$$

ce qui donne

$$
b c \square_{0}=\left(b c-f^{2}\right)\left(b c^{\prime}-b^{\prime} c\right)^{2}+\left[b\left(c f^{\prime}-c^{\prime} f\right)+c\left(b f^{\prime}-b^{\prime} f\right)\right]^{2} .
$$

En effet, en y substituant la seconde expression de $\square_{0}$, on a l'identité

$$
4 b c\left(b f^{\prime}-b^{\prime} f\right)\left(c f^{\prime}-c^{\prime} f\right)=-f^{2}\left(b c^{\prime}-b^{\prime} c\right)^{2}+\left[b\left(c f^{\prime}-c^{\prime} f\right)+c\left(b f^{\prime}-b^{\prime} f\right)\right]^{2}
$$

et l'expression pour $b c \square_{0}$ est ainsi démontrée. Mais en supposant que $(a, b, c, f, g, h)(\text {, })^{2}$ soit une forme définie, on a $b c-f^{2}=+$, donc aussi $b c=+$, et $b c \square_{0}=\left(b c-f^{2}\right) X^{2}+Y^{2}=+$, donc enfin $\square_{0}=+$. Il serait assez intéressant de trouver une démonstration pareille pour l'expression générale de $\square$.

Londres, $23^{\text {ieme }}$ Octobre 1862.

c. v. 